\title{
Study of Volume Restriction Methods Based on the Melody Structure and Vocal of Music on Portable Music Player for Hearing Protection
}

\author{
Daiki Okabe ${ }^{\mathrm{a}}$, Yoshihisa Nakatoha,* \\ ${ }^{a}$ Kyushu Institute of Technology, 1-1 Sensui-cho Tobata-ku Kitakyusyu 804-8550, City and Postcode, Japan \\ ${ }^{\mathrm{b} S}$ Second affiliation, Address, City and Postcode, Country \\ *nakatoh@ecs.kyutech.ac.jp
}

\begin{abstract}
In this paper, in order to prevent hearing loss from music listening using a portable music player, the possibility that lower the volume to a safe level without be noticed by the user (the difference of perceived attenuating level) are evaluated. According to previous studies, in the method of linearly attenuating, the notice happened when volume level was attenuated $12 \pm 4 \mathrm{~dB}$, and it not be affected by changing attenuation rate of volume level. Based on the above experiments, we examine the melody structure of music by attenuate the volume separately for melody structure (Intro part, Verse, Chorus, Interlude). As a result, it was found that the intro part was hard to notice the volume attenuation, and in the part of the chorus, it was easy to notice the volume attenuation. Next, we evaluated the difference of perceived attenuating level considering frequency characteristics. As a result for the low frequency, it was difficult to notice the volume attenuation, and the high frequency tends to notice the volume attenuation.

In this research, in order to select the optimum attenuation method, the difference of perceived attenuating level is measured by a method of attenuating while increasing and decreasing the volume. As a result, it is found that the method of linearly attenuating is optimal. Also, we compare the difference of perceived attenuating level between vocal and instrumental. As a result, it was found that instrumental is less noticeable of attenuation and the difference is about $2 \mathrm{~dB}$.
\end{abstract}

Keywords: headphone hearing loss, portable music player, hearing protection, volume attenuation, frequency, vocal.

\section{Introduction}

In recent years, portable music players have spread because of the convenience of enjoying music easily. On the other hand, it is reported that a risk of causing hearing loss is increased by listening to music at loud volume and for a long time[1,2,3]. In fact, WHO and EU have announced an indicator for listening to music safely. WHO showed people listening to music at $85 \mathrm{~dB}$ per day for one hour or more is dangerous. EU showed people listening to music at $80 \mathrm{~dB}$ per day for 5.7 hours or more is dangerous. Therefore, we propose a method to prevent hearing loss by lowering the volume so as not to notice when the user is listening to music at dangerous volume. In the following, we will describe previous research and our previous our research results. Higashi et al.[4], conducting the measurement of the difference of perceived attenuating level against the volume attenuation. Fig1 shows how the sound source attenuates. The background noise is taken from a real station because of

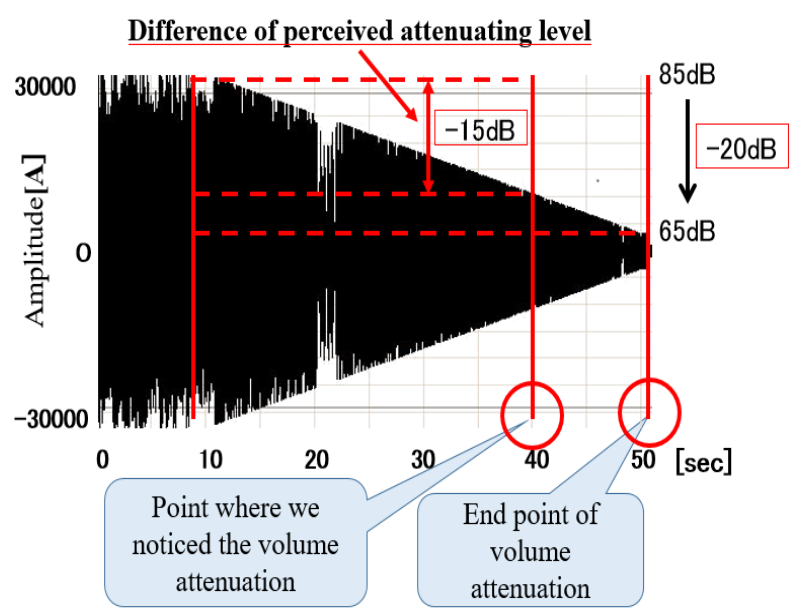

Fig1. Waveform of attenuation 
the power spectrum is available in the whole frequency range. And these music were set with the equivalent noise level of $85 \mathrm{~dB}$ as the initial value, and then the attenuation of these music were started from a certain point of these music. The ways to lower the volume were set to four kinds in total of $0.5,0.3,0.25,0.2[\mathrm{~dB} / \mathrm{sec}]$. As a result, it was known that at each attenuation rate, adults perceived in the range of about $12 \mathrm{~dB} \pm 4 \mathrm{~dB}$ from the start of the volume attenuation, and the elderly perceived in the range of about $13 \mathrm{~dB} \pm 4 \mathrm{~dB}$ from the start of the volume attenuation.

In the our previous research [5], based on the results of Higashi, in order to investigate whether it is possible to attenuate the sound volume efficiently and safely by starting the volume attenuation from where in the music, We evaluate the difference of perceived attenuating level to the structure of music. The subjects are five adult males with normal hearing ability. Of the songs used in the study of Higashi, the presented songs have selected three types of songs: Japanese lock music, Japanese punk music and Western pop music. Edited to start attenuation from four places of each song, Intro part, Verse, chorus, and interlude, unifying the decay rate to $0.5 \mathrm{~dB} / \mathrm{sec}$. Therefore, the experimental sound source is set to have four different attenuation points per song. Fig2 shows the average value and the standard deviation of the difference of perceived attenuating level with respect to the attenuation starting point. As a result, it is known that the Intro part is hard to notice the volume attenuation, and the chorus part easily notices the attenuation.

Subsequently, in order to investigate what factor in music affects the difference of perceived attenuating level, the difference of perceived attenuating level is evaluated focusing on frequency. The subjects are the same as the above-described evaluation experiments. The presentation sound source in this experiment is divided octave band from $0 \mathrm{~Hz}$ to $8 \mathrm{kHz}$, pure tones of each center frequency are created, and band tones including each center frequency are created. All attenuation rates are unified at $0.5 \mathrm{~dB} / \mathrm{sec}$. Considering the A characteristic, when the pure tone of 1 $\mathrm{kHz}$ is set to $85 \mathrm{dBA}$, the power correction is performed so as to be $85 \mathrm{dBA}$ also at the other frequency. Fig 3 shows the average value of the difference of perceived attenuating level for each frequency of pure tones and band noises. As a result, compared with the music, both pure tone and band noise tended to notice the volume attenuation easily. It is known that the higher frequency band tends to notice attenuation, and the lower frequency band tends to be less aware of attenuation.

In this research, For various investigations in the future, in order to confirm whether or not the method of linearly attenuating the volume is the optimum attenuation method, we compared it with the method of attenuating while increasing and decreasing the volume. Subsequently, we compared and evaluated the difference of perceived attenuating level for vocal and instrumental.

\section{Selection of volume attenuation method}

In this chapter, we investigate more optimal volume attenuation method.

\subsection{The proposed method}

Previous studies have evaluated only by linearly damping, but the consideration is only the decay rate, and no other attenuation methods have been investigated. Therefore, as a new attenuation method, the attenuation perception level difference is evaluated by a method of attenuating while increasing and decreasing the volume, and it is compared with the linear sound attenuation method. We thought that it

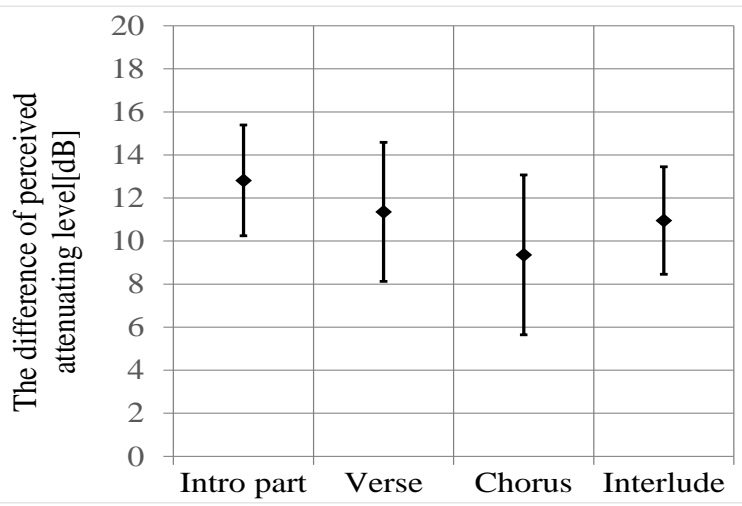

Fig2. The difference of perceived attenuating level in relation to the melody structure of music

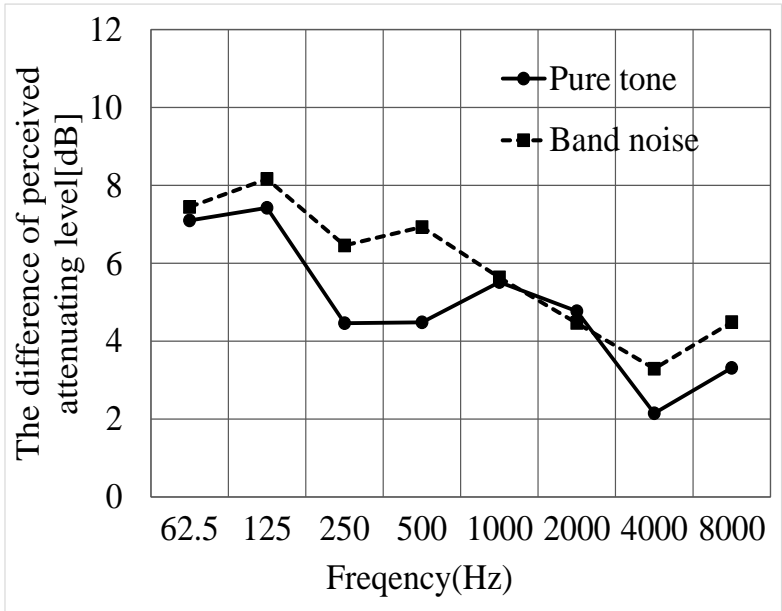

Fig3. The difference of perceived attenuating level in relation to the frequency of pure tones and band noises 
would be difficult to notice that the volume gradually decreases when increasing and decreasing the volume, so we devised this method. Fig4 shows how the sound source attenuates. A method of attenuating while increasing or decreasing the volume will be explained by extracting one of seven attenuation patterns. $4 \mathrm{~dB} / \mathrm{sec}$ reduction and $3 \mathrm{~dB} /$ $\mathrm{sec}$ rise are switched every $1 \mathrm{sec}$. This cycle is repeated until the attenuation start sound volume is attenuated by $20 \mathrm{~dB}$ from $85 \mathrm{~dB}$. This one cycle is referred to as "4-3". The attenuation patterns are seven types in total, ie, 4-3, 3-2, 2-1, $2-1.5,1.5-0.5,1.5-1$, and 1-0.5. In the method of linearly attenuating the volume, comparison is made only at $0.5 \mathrm{~dB}$ / sec. There are three types of presentation songs: Japanese rock music, Japanese punk music, Western pop music. We created a smartphone application for evaluation and played these music through the headphone connected to Apple's iPod touch with the application. In addition, the experiment was conducted in the soundproof chamber, and under the background noise in the station $(66.2 \mathrm{~dB})$ considering the situation of actually listening to the music. Subjects were 6 adult males with normal hearing from 23 to 24 years old. In addition, a questionnaire was conducted as to whether the subject experienced a sense of discomfort with the presented music so that the usefulness of the proposed method can be evaluated.

\subsection{The experimental result}

Fig5. shows the average value and standard deviation of all test subjects and all songs of the difference of perceived attenuating level for the proposed method and the conventional method. From Fig5, it was found that 4-3 was attenuated to the safest sound volume. However, in 4-3 and $3-2$, since the attenuation volume per second is too large, there were many subjects who remembered discomfort and it was considered that the usefulness was low. Therefore, the conventional method could attenuate to the safest sound volume, and the attenuation sound volume was found to be in the range of about $11 \mathrm{~dB} \pm 3 \mathrm{~dB}$. Although there was some variation in the result of each song, it was confirmed that the trend was similar to the result of the average value of all songs. Also, since the two subjects tended to notice the attenuation of the volume earlier than the other 4 subjects, the standard deviation of the results of all the songs increased slightly. These two subjects were knowledgeable in music, and since they were experienced players of musical instruments, it is thought that the sensitivity to the change in sound volume was acute. By increasing or decreasing the volume, I thought that increasing or decreasing the volume would make it hard to notice that the volume was decaying, but it was contrary to expectation. According to the questionnaire after the experiment, many opinions such as "I felt the volume fell greatly as soon as the volume decreased after increasing the volume, even if its increase and decrease width was small". Accordingly, it was considered that the subject became more aware of the volume attenuation than the conventional method. Therefore, the result that the method of attenuating the volume linearly was the optimum attenuation method than the method of attenuating while increasing and decreasing the volume was obtained.

\section{Evaluation of the difference of perceived attenuating level in relation to the vocal}

In this chapter, we investigate the influence of the vocal on the difference of perceived attenuating level.

\subsection{The proposed method}

In previous research results, it was not known whether or not vocal influences attenuation perception level difference, so we will do this experiment to explore its influence. Subjects and experimental conditions are basically the same as those in the previous chapter. The presented

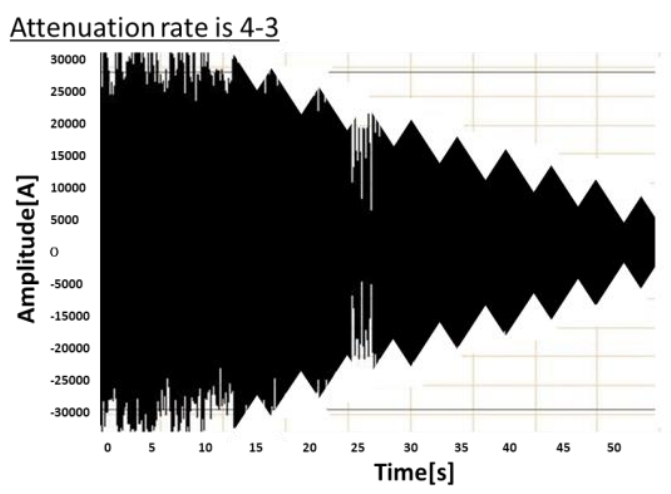

Fig4. Waveform of the new attenuation method

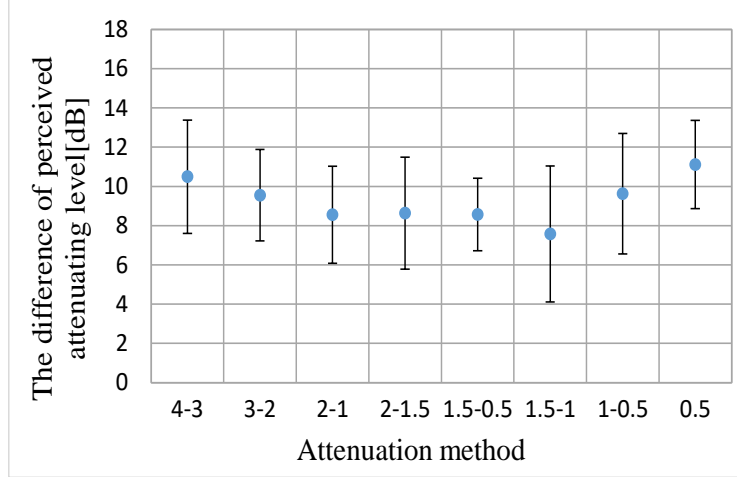

Fig5. The difference of perceived attenuating level in relation to proposed method and conventional method 
songs in this experiment are 3 songs of Japanese pop music, Japanese rock music, Western pop music. Because it was necessary to obtain experimental results with various songs, we selected songs different from the previous chapter. Sound sources of vocals and instrumentals of each song were prepared, and the attenuation rate was unified at $0.5 \mathrm{~dB} / \mathrm{sec}$. In addition, the attenuation start volume was $85 \mathrm{~dB}$, and it was set to begin attenuation from A melody.

\subsection{The experimental result}

Fig6. shows the average value and standard deviation of the difference of perceived attenuating level for vocals and instrumentals for all subjects and all songs. From Fig6, it turned out that vocals could attenuate to the safe volume than instrumentals. In addition, since the same result was obtained in all trials, it could be said that it was a highly reliable result. Instrumentals were basically the same melody in each composition such as A melody and chorus, and it was easy to notice the volume attenuation because it was easy to use the sound and melody of a specific instrument as an indicator of the volume change. Vocal was a song on which voice was on and it seems that it was difficult to notice volume attenuation because pitch and volume changes were added to the melody.

Therefore, it was found that the presence or absence of vocal influences the difference of perceived attenuating level, vocals have been attenuated to a safe volume than instrumentals. In addition, the difference of perceived attenuating level of the vocal was about $13 \mathrm{~dB}$, and the difference of perceived attenuating level of the instrumental was about $11 \mathrm{~dB}$, and the difference was $2 \mathrm{~dB}$ or more.

\section{Conclusions}

In this study, we conducted comparative evaluation of the difference of perceived attenuating level of a method of linearly attenuating the volume and a method of attenuating while increasing and decreasing the volume. As a result, it turned out that the method of linearly attenuating the volume was attenuated to a more safe volume. Moreover, investigating the influence of the presence or absence of a vocal on the attenuation perception level difference confirmed that the on-vocal was able to attenuate to a safe volume than the off-vocal, and it was confirmed that the vocal is an important element. In the future, we will investigate the relationship between background noises and the difference of perceived attenuating level and the efficient attenuation method.

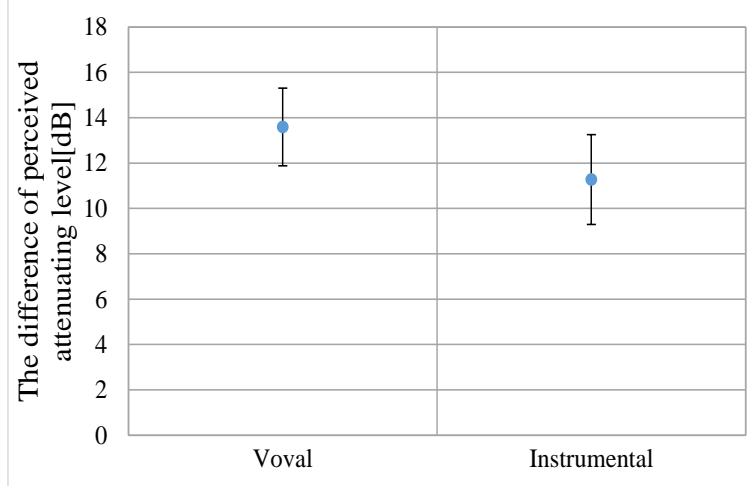

Fig6. The difference of perceived attenuating level in relation to the vocal

\section{Acknowledgment}

Part of this study, was conducted with the assistance of the Ministry of Education Science and Research Grants Fund grant (NO.24500664).

\section{References}

(1) Fumitaka Saito, Yoiti Suzuki, and Heli Laitinen, "Consideration concerning hearing of amount of exposure of sound by music and young people's hearing ", The Journal of The Acoustical Society of Japan Vol. 63 No. 4 pp233-238 (2007)

(2) Mariko Hamamura, Naoki Kishigami and Shinichiro Iwamiya, "Difference in optimum listening level for reproduced music between men and women",Journal of the Acoustical Society of Japan Vol. 70 No. 10 pp.525 533 (2015)

(3) Mariko Hamamura, Manami Aono and Shinichiro Iwamiya, "Differences in the preferred listening levels of ambient music, sound signs, public announcements, and natural environmental sounds between men and women", Journal of the Acoustical Society of Japan Vol. 71 No. 2 pp.65 -72 (2015)

(4) Teruhisa Higashi, Yuto Tanaka, and Yoshihisa Nakatoh "Study of Volume Limit for Hearing Protection in the Music Listening" IIAE CONFERENCE SYSTEM, The 3rd IIAE International Conference on Intelligent Systems and Image Processing 2015 (ICISIP2015)

(5) Daiki Okabe, Yoshihisa Nakatoh, "Study on Headphone Hearing Loss Prevention Methods Based on the Melody Structure of Music on Portable Music Player" 2018 IEEE International Conference on Consumer Electronics (ICCE) 